Review

\title{
Topical Peptide Treatments with Effective Anti-Aging Results
}

\author{
Silke Karin Schagen \\ Beldio Research GmbH; Kramerstrasse 15, 87700 Memmingen, Germany; info@beldio.com \\ Academic Editor: Marie Loden \\ Received: 26 December 2016; Accepted: 16 May 2017; Published: 22 May 2017
}

\begin{abstract}
In the last two decades, many new peptides have been developed, and new knowledge on how peptides improve the skin has been uncovered. The spectrum of peptides in the field of cosmetics is continuously growing. This review summarizes some of the effective data on cosmeceutical peptides that work against intrinsic and extrinsic aging. Some peptides have been proven in their efficacy through clinical skin trials. Well-known and documented peptides like copper tripeptide are still under research to obtain more details on their effectiveness, and for the development of new treatments. Palmitoyl pentapeptide- 4 and Carnosine are other well-researched cosmeceuticals. Additionally, there are many more peptides that are used in cosmetics. However, study results for some are sparse, or have not been published in scientific journals. This article summarizes topical peptides with proven efficacy in controlled in vivo studies.
\end{abstract}

Keywords: barrier function; anti-aging; skin care; peptides; clinical studies

\section{Introduction}

Peptides and proteins are amino acid polymers. Peptides are short amino acid chains. The name peptide is coming from pepton [peptos: digested (Greek)]. Naturally occurring human peptides are known for cellular communication, such as protein regulation, cell proliferation, cell migration, inflammation, angiogenesis, and melanogenesis [1], which results in a broad variety of physiological processes including defense, immunity, stress, growth, homeostasis, and reproduction [2]. The first peptides were described by Emil Fischer and Hofmeister in the early 19th century. The first peptide synthesis was published in 1901 by Fischer and Fourneauin [3]. Fischer described the first peptide as a glycyl-glycine and in his lectures explained more peptide structure like dipeptides, tripeptides and polypeptides [4]. Years followed, and scientists synthesized new peptides, identified more natural peptides, and learned more about their functions. Beside the growing knowledge about natural and synthetic peptides, different synthetic peptides were developed. Copper glycine-histidine-lysine (Cu-GHK) was developed in 1973 by Loren Pickard. In the late 80s, the first copper peptide was incorporated into skin care products. Even then, peptide development proceeded slowly until the beginning of 2000, when palmitoyl pentapeptide- 4 was established. Since then, research and industry has developed many short, stable, and synthetic peptides that have a role in extracellular matrix synthesis, pigmentation, innate immunity and inflammation. These peptides are used for collagen stimulation, wound healing, "Botox-like" wrinkle smoothing, as well as antioxidative, antimicrobial [5], and whitening effects.

Topical cosmeceutical peptides can be classified as signal peptides, carrier peptides, neurotransmitter inhibitor peptides, and enzyme inhibitor peptides.

Cosmeceutical peptides should have certain features in order to obtain good effects. Historically, it has always been assumed that because of the skin barrier, the molecular weight of peptides should be less than $500 \mathrm{Da}$, otherwise the peptide would not be able to pass the barrier. The moderate log 
of the partition coefficient for octanol/water should be between 1 and 3, the melting point should be below $200{ }^{\circ} \mathrm{C}$, water solubility should be $>1 \mathrm{mg} / \mathrm{mL}$ and there should be no or few polar centers [6].

Newer studies have shown that larger molecules can traverse the skin barrier, especially in the case of dry and aged skin [7,8]. Synthetic peptides consist of amino acids chains which can be now be modified in various ways for different functions like increased skin penetration, and increased special receptor binding, stability, and solubility.

Some peptides are used in cosmetic products as shown in Table 1, but little in vivo efficacy data is available [2,6]. In addition, substance mixtures are on the market and tested in cosmetic formulations, so that in many cases the actual effect of individual peptides on the skin remains unclear. Claims of efficiency by cosmeceuticals are restricted to the improvement of the skin appearance. Improving cosmeceutical function might lead to re-classification from cosmetic to drug category, which is often not desirable. This often limits possibilities for development.

Research on peptides should aim to identify the peptide's mode of action, and define it for cosmetic and/or pharmaceutical use. The prerequisites for an effective active substance must be carefully examined before it is used. Study designs should be developed carefully, and maximal results should be generated. With today's methods, receptor activation, efficacy, and mechanistic information can be identified. Interesting and meaningful in vivo studies can be developed. The development of active peptides has opened a new field in cosmeceutical and pharmaceutical skin care in the last decade. This review summarizes clinical studies with peptides which confirm their efficacy on human skin.

Table 1. Topically used peptides.

\begin{tabular}{cl}
\hline Type of Peptide & \multicolumn{1}{c}{ Name of Peptides } \\
\hline Matricins peptides & $\begin{array}{l}\text { Carnosine, Copper tripeptide, Trifluoroacetyl-tripeptide-2, } \\
\text { Tripeptide-10 citrulline, Acetyl tetrapeptide-5, Acetyl tetrapeptide-9, } \\
\text { Acetyl tetrapeptide-11, Tetrapeptide PKEK, Tetrapeptide-21, } \\
\text { Hexapeptide, Hexapeptide-11, Palmitoyl pentapeptide-4, Palmitoyl } \\
\text { tripeptide-3/5, Palmitoyl tetrapeptide-7, Palmitoyl hexapeptide-12, } \\
\text { Palmitoyl oligopeptide, Palmitoyl tripeptide-1, Pentamide-6 }\end{array}$ \\
\hline Carrier peptides & Copper tripeptide, Manganese tripeptide-1 \\
\hline Peptide mimetics or & Acetyl hexapeptide-3, Pentapeptide-18, Pentapeptide-3, Tripeptide-3 \\
\hline neurotransmitter-inhibiting peptides & Soybean peptide, Silk fibroin peptide, Black rice oligopeptides \\
\hline Structural protein digestion & Keratin peptide \\
\hline
\end{tabular}

\section{Peptides that Trigger the Signaling Cascade}

A number of peptides are able to trigger a signaling cascade. They are released from the extracellular matrix, and are also called matricins or collagen stimulators. With these peptides, the proliferation of collagen, elastin, proteoglycan, glycosaminoglycan and fibronectin is increased. As a consequence, pigmentation of photo-damaged skin and fine lines and wrinkles are reduced with the regeneration of the skin matrix cells. Skin elasticity increases, and skin appears smoother and firmer. Synthetic peptides modeled on repair signaling sequences like the following described in this section, have been developed to rejuvenate skin.

\subsection{Carnosine and N-Acetylcarnosine}

Carnosine is a dipeptide (Sequence: $\beta$-Ala-His) and a well-documented aqueous antioxidant with wound healing activity, and it is naturally present in high concentrations in muscle and brain tissues. Carnosine is a scavenging reactive oxygen species as well as an $\alpha-\beta$ unsaturated aldehyde formed from peroxidation of cell membrane fatty acids during oxidative stress. The low molecular weight water soluble unmodified dipeptide $\beta$-Ala-His has very little affinity for skin and does not penetrate beyond the first layer of the stratum corneum. The lipophilic peptide palmitoyl $\beta$-Ala-His however, 
diffuses into the stratum corneum, epidermal, and dermal skin layers. No systemic activity has been observed [9].

In two double-blind, randomized, controlled, split-face studies of four weeks each, changes in periorbital wrinkles in women (aged 30-70) were observed (Study 1, 42 volunteers; Study 2, 35 volunteers). Tested products containing niacinamide, the peptides pal-KT and pal-KTTKS, and carnosine, ameliorated periorbital skin, enhancing smoothness and diminishing larger wrinkle depth [10].

A double-blind irradiation study comparing a complex consisting of different active ingredients (SPF 50, photolyase, endonuclease, 8-oxoguanine glycosylase, carnosine, arazine, and ergothionine) in available products with DNA repair, antioxidant and growth factor ingredients, found the formulation to be effective in reducing pyrimidine dimers, protein carbonylation, and 8-oxo-7,8-dihydro-2'-deoxyguanosine in human skin biopsies. The formulation also appeared to enhance the genomic and proteomic integrity of skin cells after continual UV exposure. Hence, this formulation could be regarded as potentially lowering the risk of UV-induced cutaneous aging, and non-melanoma skin cancer [11].

During a six-month study, 20 healthy volunteers (Photo type II or III) were treated with carnosine and $\mathrm{N}$-acetylcarnosine formulations. Carnosine and $\mathrm{N}$-acetylcarnosine alone in a water solution obtained $3.6 \%$ and $7.3 \%$ reduction of erythema compared to the control. Both peptides showed antioxidant capacity, with a higher significance in conjunction with vehicles improving the substances' skin penetration capabilities. $\mathrm{N}$-acetylcarnosine was mentioned as an interesting hydrophilic antioxidant for dermatological purposes [12].

\subsection{Trifluoroacetyl-Tripeptide-2}

Trifluoroacetyl-tripeptide-2 (Sequence: TFA-Val-Try-Val-OH) was evaluated in two in vivo split face studies. One study examined its anti-wrinkle and anti-sagging effects along the jawline of 10 volunteers (56 days) via fringe projection profilometry; and the other study targeted skin firmness, elasticity, and viscoelasticity via cytometry on 13 healthy volunteers (28 days). According to the studies, trifluoroacetyl-tripeptide-2 has progressive effects on wrinkles, firmness, elasticity and sagging [13].

\subsection{Tripeptide-10 Citrulline}

Tripeptide-10 citrulline (Sequence: Lys- $\alpha$-Asp-Ile-Citrulline), a decorin-like tetrapeptide, Decorinyl $^{\mathrm{TM}}$ is used to specifically target collagen fiber organization. Puig et al. published results of an assessor blinded, placebo-controlled, parallel group study with 43 healthy volunteers (aged 40-58). Tripeptide-10 citrulline showed uniformity in fibril diameter, and increased skin suppleness from better collagen fiber cohesion [14].

\subsection{Palmitoyl Tripeptide-1}

Palmitoyl tripeptide-1, also called pal-GHK and palmitoyl oligopeptide (Sequence: Pal-Gly-His-Lys), is a messenger peptide for collagen renewal. Comparable to retinoic acid with regards to its activity, it does not trigger irritation. Collagen and glycosaminoglycan synthesis are stimulated, the epidermis is reinforced, and wrinkles are diminished. This peptide is suggested to act on TGF $\beta$ to stimulate fibrillogenesis. It is used in cosmetic anti-wrinkle skincare and make-up products. In a study with 15 women, a cream containing palmitoyl tripeptide- 1 was applied twice daily for four weeks, leading to statistically significant reductions in wrinkle length, depth and skin roughness. Another study applied both vehicle and palmitoyl tripeptide- 1 to the skin of 23 healthy female volunteers for four weeks [9], documenting a small but statistically significant increase in skin thickness ( 4\%, compared to the vehicle alone) [15].

A combination of pal-GHK tripeptide and pal-GQPR tetrapeptide is marketed as an anti-wrinkle compound with the trade name Matrixyl ${ }^{\mathrm{TM}} 3000$. A blind, randomized clinical study with 28 volunteers twice daily applying cream including the active compound to half their face and one of their forearms 
and a placebo cream to the other half of the face and other forearm confirmed anti-wrinkle efficacy, reduction of wrinkle depth, volume and density, skin roughness and complexity, as well as a decrease of the area occupied by deep wrinkles, and an increase in skin tone [16].

\subsection{Palmitoyl Tripeptide-3/5}

Palmitoyl tripeptide-3/5 mimics the effects of an extracellular matrix protein, thrombospondin-1 (TSP-1), a naturally occurring molecule that increases TGF $\beta$ activity. In animal models and human dermal fibroblasts cell culture tests, TSP-1 acts locally to improve wound healing, and is believed to be active in the post-natal development of skin structures [17]. The short sequence Lys-Arg-Phe-Lys of the TSP-1 protein is responsible for TGF $\beta$ stimulation. TGF $\beta$ in turn, causes a persistent increase in the amounts of Type I and Type III collagen that dermal fibroblasts produce. Palmitoyl tripeptide-3/5 (Sequence: Pal-Lys-Val-Lys bistrifluoracetae salt) $\left(S Y N^{\circledR}\right.$-COLL) stimulates collagen production in in vitro and in vivo studies through the growth factor TGF $\beta$.

Animal studies indicate that palmitoyl tripeptide-3/5 may increase collagen synthesis. In vitro studies show that palmitoyl tripeptide-3/5 can prevent collagen breakdown by interfering with MMP1 and MMP3 collagen degradation. Palmitoyl tripeptide-3/5 seems to boost collagen synthesis, but decreases collagen breakdown. Additional data show that palmitoyl tripeptide-3/5 is roughly 3.5 times more effective at reducing the appearance of wrinkles than the placebo [5]. In vivo results of a palmitoyl tripeptide-3/5 (10 to $25 \mathrm{ppm}$ ) cream formulation demonstrated a dose-dependent wrinkle reduction, measured by PRIMOS surface topography [18].

In an efficacy study performed on 60 Chinese volunteers (84 days, applied twice daily), palmitoyl tripeptide-3/5 confirmed its anti-wrinkle efficacy and reduced skin roughness better than control groups, placebo and pal-KTTKS-containing creams [6].

\subsection{Palmitoyl Tripeptide-38}

Epithelial regeneration in skin is achieved by the constant turnover and differentiation of keratinocytes. Epidermal and dermal stem cell compartments are fundamental for the continuous renewal of the skin. Adult stem cells are the unique source for skin tissue renewal. Plants also have stem cells and plant-derived stem cell extracts are now used in topical products for their potential anti-aging and anti-wrinkle effects.

A dermocosmetic product containing apple stem cell extract, urea, creatine and palmitoyl tripeptide-38 (Sequence: Pal-Lys-Met(O2)-Lys-OH) was applied on the face twice daily for 28 days, and assessed by clinical and instrumental evaluation in 32 women with sensitive skin bearing crow's feet wrinkles. The treatment results showed a significant increase in dermal density and elasticity, as well as anti-wrinkle effects. The anti-aging serum seems to improve aging skin signs with the first visible results achieved after one week treatment [19].

To determine the effectiveness of a multi-ingredient anti-aging moisturizer, an open label clinical trial was conducted with 37 female subjects of ages 35-60. The effective ingredients of the moisturizer for the facial skin included Astragalus membranaceus root extract, a peptide blend including palmitoyl tripeptide-38, standardized rosemary leaf extract (ursolic acid), tetrahexyldecyl ascorbate, and ubiquinone. Results were favorable in both product efficacy measurements and aesthetic self-assessment questionnaires, with subjects judging the product as being mild and well-tolerated [20].

\subsection{Palmitoyl Pentapeptide-4}

Palmitoyl pentapeptide-4, (Matrixyl ${ }^{\circledR}$ ) (Sequence: Pal-Lys-Thr-Thr-Lys-Ser-OH or pal-KTTKS-OH) is a small, highly specific biologically active peptide which has been reported to stimulate the production of elastin, fibronectin, glucosaminoglycan and collagens (specifically Types I, III and IV), support of the extracellular matrix, and wound healing. KTTKS structure is related to the precursor of collagen Type I (or procollagen Type I). The stimulatory effect of KTTKS on collagen Types I and III, and fibronectin, seems to relate mainly to the biosynthetic pathway, rather than the 
export or degradation pathways. KTTKS has a molecular weight of $563.64 \mathrm{Da}$, the longer pal-KTTKS is 802.05 Da.

In a placebo-controlled double blind study, pal-KTTKS (0.005\%) formulation was applied to the right periocular area twice daily for 28 days [21]. As demonstrated by optical profilometry, this resulted in a quantitative decrease in fold depth, fold thickness, and skin rigidity, by $18 \%, 37 \%$, and $21 \%$, respectively. These results were confirmed in two other placebo-controlled double blind studies in women (42 and 35 subjects) with moderate to distinct periorbital wrinkles [10].

A double-blind, placebo-controlled, split face, left-right randomized trial involving 93 subjects was carried out to assess the clinical efficacy of pal-KTTKS, with fine line or wrinkle improvement as the parameter of interest [22]. In another four month-long double-blind study, 49 women were directed to apply either pal-KTTKS or vehicle twice daily to their faces. The results showed that pal-KTTKS exhibited significant improvement in skin roughness, wrinkle volume, and wrinkle depth, compared with the vehicle. Data associated pal-KTTKS with an increase in elastin fiber density and thickness, as well as improved collagen IV regulation at the dermal-epidermal junction [23].

\subsection{Palmitoyl Tetrapeptid-7}

Palmitoyl tetrapeptide-7 (Rigin ${ }^{\mathrm{TM}}$ ) (Sequence: Pal-Gly-Gln-Pro-Arg or pal-GQPR) is a fragment of immunoglobulin G. Palmitoyl tetrapeptide-7 decreases IL-6 secretion in a basal setting, and serves as an anti-inflammatory after exposure to UVB-irradiation. In vivo reflectance confocal microscopy studies indicated that a blend of palmitoyl oligopeptide and palmitoyl tetrapeptide-7 enhanced the extracellular matrix structure compared to placebo [24]. Sixty healthy photoaged volunteers (aged 45-80) were tested over 12 months with a formulation containing palmitoyl tetrapeptide-7 and another active ingredient. A reduction of facial wrinkles was documented by this long-term use. Better skin appearance was related to the deposition of fibrillin-rich microfibrils in the papillary dermis of treated skin [25].

\subsection{Palmitoyl Hexapeptide-12}

Palmitoyl hexapeptide-12 (Sequence: Pal-Val-Gly-Val-Ala-Pro-Gly), which is the peptide in Biopeptide-EL, creates a response in the dermis of the skin that stimulates collagen and elastin fibroblasts, developing fibronectin and glycosaminoglycans. It is believed to work by reducing the production of interleukin-6 (IL-6) by key skin cells, keratinocytes and fibroblasts. IL-6 is a molecule that promotes inflammation, which, in turn, leads to faster degradation of the skin matrix, and thus contributes to the development of wrinkles, and loss of skin firmness and elasticity. By reducing the levels of IL-6 and possibly other inflammation mediators, palmitoyl is thought to slow down the degradation of the skin matrix, and may also stimulate its replenishment.

A one month double-blind study was conducted on 10 female volunteers, aged 32-56, who performed twice daily applications of a light emulsion containing $4 \%$, or a placebo. Palmitoyl hexapeptide treatment improved elasticity, tone, skin fatigue and firmness [26].

\subsection{Acetyl Tetrapeptide-9/11}

Acetyl tetrapeptide-9 (Dermican ${ }^{\mathrm{TM}}$ ) (Sequence: $N$-Acetyl-Gln-Asp-Val-His) is reported to stimulate collagen Type I and lumican synthesis, whereas acetyl tetrapeptide-11 (Sequence: $N$-Acetyl-Pro-Pro-Tyr-Leu) (Syniorage ${ }^{\mathrm{TM}}$ ) stimulates keratinocyte cell growth and syndecan-1 synthesis . Clinical studies documented that treatment with acetyl tetrapeptide-9 (17 female volunteers) or acetyl tetrapeptide-11 (19 female volunteers) led to thicker and firmer skin. Both peptides were more effective compared to placebos [27].

\subsection{Tetrapeptide-21}

Tetrapeptide-21, also named GEKG (Sequence: Gly-Glu-Lys-Gly), was derived from ECM proteins. Its amino acid sequence is glycine-glutamic acid-lysine-glycine. This peptide demonstrated in 
in vitro studies, an increase of collagen (Type I) production on the protein level and mRNA level, hyaluronic acid synthase 1 production, and a strong increase in fibronectin (GEKG Conc. 0.001\%). Increase in collagen (COL1A1), procollagen, hyaluronic acid and fibronectin, as well as skin elasticity, were measured in a double-blind, randomized, placebo-controlled study (10 women/eight weeks). A placebo-controlled study with 30 subjects was carried out to analyze the effect of GEKG on facial wrinkles. GEKG significantly decreased skin roughness.

In a comparison study with GEKG vs. pal-KTTKS with 60 subjects, elasticity increased after two daily treatments over eight weeks by $41.3 \%$ for GEKG, while pal-KTTKS showed an improvement of $35.6 \%$ [28].

\subsection{Tetrapeptide PKEK}

Tetrapeptide PKEK (Sequence: Pro-Lys-Glu-Lys) can exert skin whitening effects. For PKEK development, Lys-Glu-Lys (KEK) was modified with proline to stabilize the peptide structure. Study results showed that PKEK reduces interleukin-6, interleukin-8, and tumor necrosis factor- $\alpha$, as well as cyclooxygenase gene expression in UV light-stressed keratinocytes [29].

The treatment of human keratinocytes with PKEK significantly reduced UVB-stimulated mRNA expression of interleukin (IL)-6, IL-8 and TNF- $\alpha$ and, most importantly, proopiomelanocorticotropin (POMC). In a randomized, double-blind, vehicle-controlled study with PKEK treatment once daily, punch biopsies of 10 healthy volunteers were taken after four weeks. PKEK treatment significantly inhibited UVB-induced upregulation of genes encoding for IL-1 $\alpha$, IL-6, IL-8, TNF- $\alpha$ as well as POMC and tyrosinase in skin areas pretreated with PKEK.

A second study was performed as a half-face in vivo efficacy test with 39 Caucasian women. Facial pigment spots were significantly faded after six weeks when PKEK was combined with the skin whitener sodium ascorbyl phosphate. PKEK or sodium ascorbyl phosphate alone led to less pronounced fading of the pigment spots than a combination of the two. An in vivo hand cream efficacy study confirmed the above result after application for eight weeks to the back of the hands of 19 Caucasians.

In the fourth study, 27 Japanese women were separated in two groups which treated faces twice daily with either sodium ascorbyl phosphate only, or with a PKEK + sodium ascorbyl phosphate formulation, for eight weeks. Application of PKEK + sodium ascorbyl phosphate significantly reduced skin pigmentation by $26 \%$, and sodium ascorbyl phosphate by $18 \%$, according to the SCINEXA score. It was confirmed that PKEK has the capacity to reduce UVB-induced skin pigmentation in all study models, and it may be suitable as a skin tone-modulating agent in cosmetic products [30].

\subsection{Hexapeptide-11}

Hexapeptide-11 (Pentamide-6, Sequence: Phe-Val-Ala-Pro-Phe-Pro) was originally isolated from Saccharomyces yeast fermentation, but was later synthesized because of purity issues. Hexapeptide-11 has the ability to influence the onset of senescence in intrinsically and extrinsically aged fibroblasts, and extrinsically aged dermal papillae cells in vitro [31]. Gorouhi and Maibach reviewed a placebo-controlled study with 25 healthy volunteers, treating their skin twice daily for four weeks. Initial skin elasticity and deformation response were improved [6].

\subsection{Hexapeptide-14}

Hexapeptide-14 (palmitoyl hexapeptide-14) has been reported to stimulate cell migration, collagen synthesis, and fibroblast proliferation and scaffolding [1]. A 12-week study with 29 volunteers showed results of reduced fine lines and wrinkles by palmitoyl hexapeptide-14. Results were compared with tretinoin and no irritations were noted [6]. 


\section{Carrier Peptides}

Carrier peptides deliver or stabilize trace elements like copper and manganese, necessary for wound healing and enzymatic progress. These peptides are involved in copper or manganese transport into skin cells. Additionally, they are obtained by binding copper with a tripeptide [32].

\subsection{Copper Tripeptide}

Copper tripeptide (Cu-GHK, lamin ${ }^{\circledR}$ ) complex (Sequence: Copper Gly-L-His-L-Lys) is one of the most well-examined peptides. It plays a role in the extracellular matrix, and is released in wounds or inflammation to support healing. It acts as signal and carrier peptide, promotes regular collagen, elastin, proteoglycan, and glycosaminoglycan synthesis, and provides anti-inflammatory and antioxidant responses. In cosmetic applications, Cu-GHK is used in anti-aging, anti-wrinkle, after-sun, skin renewal, skin moisturizer, hair growth stimulating products [33].

$\mathrm{Cu}-\mathrm{GHK}$ stimulates cellular regulatory molecules and regenerates, and heals skin and other tissues. Stem cells treated with GHK regenerated and expressed more stem cell markers. GHK and Gly-Gly-His (GGH) reduce TNF- $\alpha$ induced cytokines IL-6 [34], thus ensuring better wound healing. Pickart et al. described that GHK significantly increased the expression of DNA repair genes, while 47 genes are stimulated and five genes are suppressed [35]. GHK is involved with different mechanisms of action and can apparently promote regeneration, healing, and repair. It furthermore achieves good effects against the aging processes.

$\mathrm{Cu}-\mathrm{GHK}$ can stimulate hair growth. The copper tripeptide complex ensures follicular enlargement and helps covering the follicle with a downy hair; the effect is comparable with that of Minoxidil [36]. The results of a hair transplant showed significant improvement [37] following application with a copper tripeptide product. Topical Cu-GHK products stimulate collagen synthesis on the scalp, strengthen existing hair, and encourage hair growth [38].

Several studies confirming Cu-GHK efficacy in various areas have been performed. These include: increasing keratinocyte proliferation, improving appearance, firmness, elasticity, skin thickness, wrinkles, spotty hyperpigmentation and light damage, skin collagen, strengthening proteins of skin protection barrier, and improvement of skin appearance.

Experiments have shown that in vitro Cu-GHK increases and stimulates the synthesis of collagen, glycosaminoglycans and other extracellular matrix molecules. Several placebo-controlled clinical trials have confirmed the observed effects [37]. A topically applied cream with Cu-GHK was shown to stimulate dermal skin procollagen synthesis. Synthesis induced by the copper tripeptide was significantly superior to vitamin C, tretinoin, or melatonin in comparison.

Similarly, a study of 20 women compared the skin's production of collagen after applying creams containing $\mathrm{Cu}-\mathrm{GHK}$, vitamin $\mathrm{C}$, or retinoic acid to thighs daily for one month. New collagen production was determined by skin biopsy samples using immunohistological techniques. After one month, Cu-GHK increased collagen in $70 \%$ of those treated, versus $50 \%$ treated with vitamin C, and $40 \%$ treated with retinoic acid [39].

Leyden et al. confirmed in two different studies (12 weeks of application by 71 or 41 women) the clinically beneficial effects of $\mathrm{Cu}-\mathrm{GHK}$ formulations on both aged and sun damaged skin. The Cu-GHK face cream and an eye cream reduced the visible signs of skin aging and caused an increase in skin density and thickness. The researchers observed improved skin elasticity and skin humidity, significant smoothing of the skin by stimulating the synthesis of collagen, a significant improvement of the skin contrast, and diminished wrinkles [36].

In another study $\mathrm{Cu}-\mathrm{GHK}$ cream was applied twice a day for 12 weeks on 67 women. The $\mathrm{Cu}-\mathrm{GHK}$ cream improved the appearance of photo-damaged, aged skin. By histological analyses of biopsies, it was re-confirmed that the use of topically applied $\mathrm{Cu}-\mathrm{GHK}$ products intensified skin thickness in the range of the epidermis and dermis, and that keratinocyte proliferation of the skin was greatly stimulated [40]. 


\subsection{Manganese Tripeptide-1}

Besides the well-researched copper tripeptide, there is also one clinical study focusing on manganese tripeptide- 1 (Sequence: GHK- $\mathrm{Mn}^{2+}$ ). This study evaluated the effects of a manganese peptide complex in the treatment of various signs of cutaneous facial photo-damage. During a 12-week period, subjects were instructed to apply a facial serum formulation containing the manganese peptide complex twice a day. This resulted in their skin's photo-damage ranking shifting from moderate to mild. The most significantly improved parameters were associated with hyperpigmentation, while no significant cutaneous inflammation was reported [41].

\section{Neurotransmitter Inhibitor Peptides}

Muscle contraction is another strategy to reduce common ageing signs like fine lines and wrinkles. Muscles are contracted by neurotransmitter release from neurons. The neurotransmitter begins a cascade with protein-protein interactions that culminate in the fusion of neurotransmitter loaded vesicles with the neuron membrane. A local modification in the membrane potential triggers the entry of calcium ions into the neuron via the terminal. When these ions enter the pre-synaptic terminal, vesicles containing acetylcholine join other parts of the neuron to release acetylcholine. This process is mediated by a SNAP receptor protein, which include the vesicle-associated membrane protein, the membrane-associated protein syntaxin, and synaptosomal-associated protein 25 . These proteins directly manage vesicle docking and fusion though the formation of the SNARE complex. The SNARE complex captures vesicles and fuses them with the membrane. Once the fusion of these vesicles occurs, acetylcholine is released into the synapse between muscle and nerve. Acetylcholine binds to acetylcholine receptors located on the surface of the muscle cells, and this leads to muscle contraction.

Topical synthetic peptides which imitate the amino acid sequence of the synaptic protein SNAP-25 were shown to be specific inhibitors of the neurosecretion. For this reason, these peptides are also called neurotransmitter inhibitor peptides. These cosmeceutical peptides penetrate skin and relax muscles, causing the reduction and softening of wrinkles and fine lines.

\subsection{Acetylhexapeptide-3}

Acetylhexapeptide-3 (Argireline ${ }^{\circledR}$ ) has the sequence Acetyl-Glu-Glu-Met-Gln-Arg-Arg- $\mathrm{NH}_{2}$, and is reported to inhibit the release of neurotransmitters, which is followed by anti-wrinkle, moisturizing effects. It seems to improve the firmness and tone of the skin. Acetylhexapeptide- 3 is a copy of the synaptosomal-associated protein 25 , which competes for a position in the SNARE complex, and destabilizes its formation without breaking any of its constituent parts. Additionally, it inhibits catecholamine secretion [42]. An increase in peptide concentration and the permeation of peptides, and a decrease in iontophoretic permeability coefficients, are affected by a number of parameters that can be optimized for effective transdermal peptide delivery [43].

Blanes-Mira described a placebo-controlled study in which acetylhexapeptide- $3(10 \%)$ vs. placebo creams were applied twice daily (10 women, 30 days). Acetylhexapeptide-3-treated skin areas showed a $30 \%$ improvement in wrinkles in the eye area [44]. The anti-wrinkle efficacy in the acetylhexapeptide-3 group was measured as $48.9 \%$, compared with $0 \%$ in the placebo group. All parameters of roughness were decreased in the same study. The fold depth in the acetylhexapeptide- 3 group (48.9\%) was significantly reduced (60 subjects) [45].

\subsection{Pentapeptide-3}

Pentapeptide-3 $\left(\right.$ Vialox $^{\circledR}$ ) (Sequence: Gly-Pro-Arg-Pro-Ala) is derived from snake venom. It is an antagonist of the acetylcholine receptor, and blocks nerves at the post-synaptic membrane, leading to muscle relaxation. Clinical studies demonstrated a reduction of wrinkles by $49 \%$, and lesser skin roughness (47\%) after a treatment of 28 days [46]. 


\subsection{Pentapeptide-18}

Pentapeptide-18 (Leuphasyl ${ }^{\circledR}$ ) (Sequence: Tyr-D-Ala-Gly-Phe-Leu) mimics the natural mechanism of enkephalins, and inhibits neuronal activity and catecholamine release. Its action can be described as having botox-like effects; and it demonstrates a proven efficacy for reducing fine lines and wrinkles, moisturizing the skin, and improving firmness and skin tone.

A cream with pentapeptide-18 (0.05\%) was compared in a study (43 women) with acetylhexapeptide-3 (0.05\%) and the combination of both peptides. Wrinkle reductions were measured for pentapeptide-18, acetylhexapeptide-3, and a combination, at $11.64 \%, 16.26 \%$, and $24.62 \%$, respectively. The pentapeptide-18 study mentioned a synergistic effect or an increase of efficiency, by its association with acetylhexapeptide-3 [6,47]. The cellular pathway of both peptides is different.

Three concentrations $(0.5 \%, 1 \%$, and $2 \%)$ of Leuphasyl formulations were applied on 20 volunteers for two months, at the level of mimic muscles in the eyebrows zone and in the periorbital zone [48]. To further evaluate anti-wrinkle effects, an in vivo study with 22 females with an average age of 51, was designed. Volunteers were injected botulinum toxin in the periorbital region combined with a topical treatment twice daily for six months. In comparison with the control, acetyl hexapeptide- 8 and pentapeptide-18 induced a $20 \%$ and $11 \%$ decrease on glutamate release, respectively, while their combination caused a $40 \%$ reduction. In addition to the measured effects of each individual compound, this indicates a synergistic activity. The topical treatment demonstrated a potentiated effect for botulinum toxin treatment, and extended the anti-wrinkle benefit on the skin [49].

\subsection{Tripeptide-3}

Tripeptide-3 (Sequence: $\beta$-Ala-Pro-Dab-NHBn-2-Acetate), also named dipeptide diaminobutyroyl benzylamide diacetate or $S Y N^{\circledR}-A K E$, mimics the effect of waglerin-1, a peptide that is found in the venom of the viper Tropidolaemus wagleri. Tripeptide-3 acts at the postsynaptic membrane, and is a reversible antagonist of the acetylcholine receptor.

Tripeptide- 3 has been tested in different preparations for topical application in animal models in concentrations ranging from $1 \%$ to $4 \%$. The results have been claimed as smoothing the appearance of mimic wrinkles and expression lines, shortly after applying the preparation [50]. Research results show that tripeptide- 3 can reduce the appearance of wrinkles after 28 days by up to $52 \%$ when a $4 \%$ topical solution is used [46]. The results from a three-month study (37 female volunteers) demonstrated that tripeptide- 3 formulation treatment provided both immediate and long-term improvements in the appearance of fine and coarse wrinkles [51].

\section{Enzyme Inhibitor Peptides}

Enzyme inhibitor peptides directly or indirectly inhibit enzymes. Soy oligopeptides, silk fibroin peptide and rice peptides act on the skin cells. The above-mentioned peptides inhibit enzymes like tTAT-superoxide dismutase, stimulate hyaluronan synthase 2, or in the case of soy oligopeptides, inhibit proteinases.

This category of peptides shows promising results, however only very few or no in vivo studies have been conducted. Hence, their relevance is still unclear.

\subsection{Soybean Peptides}

Soy oligopeptides are obtained from soybean proteins, consisting of 3-6 amino acids, mainly in the size range of 300-700 kDa. Various biological activities of soybean oligopeptides have been identified, such as antioxidant, blood pressure lowering, and blood lipid lowering effects. Topically applied soy oligopeptides data showed significantly increased Bcl-2 protein expression and decreased cyclobutane pyrimidine dimers-positive cells, sunburn cells, apoptotic cells, p53 protein expression, and Bax protein expressions in the epidermis of UVB-irradiated foreskin. Topically used soy oligopeptides seem to protect human skin (nine healthy male volunteers) against UVB-induced photo damage [52]. 
A pseudo-randomized study (10 women, in vivo/in vitro) with soybean peptide showed a significant increase in glycosaminoglycan and collagen synthesis. This study showed the anti-aging potential of a soy peptide [53].

\subsection{Silk Fibroin Peptide}

Silk fibroin peptide is derived from the silkworm Bombyx mori. Scientific results show inhibitory inflammation and enhance the anti-inflammatory activity of tTAT-superoxide dismutase, which was previously reported to effectively penetrate various cells and tissues, and exert anti-oxidative activity in a mouse model of inflammation [54]. No human in vivo efficacy data are currently available.

\subsection{Rice Peptides}

After a specially processing rice bran protein, low molecular weight peptides (<3000 Da) were obtained. Black rice oligopeptides were measured at approximately $1300 \mathrm{Da}$. These oligopeptides were noted for inhibiting MMP (matrix metalloproteinase) activity and stimulated hyaluronan synthase 2 gene expression in human keratinocytes in a dose-dependent manner [55]. Three new identified rice bran protein peptides had a C-terminal tyrosine residue, and exhibited significant inhibitory effects against tyrosinase-mediated monophenolase reactions. Additionally, one peptide called CT-2 (Leu-Gln-Pro-Ser-His-Tyr) potently inhibited melanogenesis in mouse melanoma cells without causing cytotoxicity, which might be of interest for melanin-related skin conditions.

Rice bran protein is a potent source of tyrosinase inhibitory peptides [56]. Formulations containing niosomes entrapped with rice bran bioactive compounds show clinical anti-aging properties [57]. However, there is currently no in vivo data for purified black rice oligopeptides available.

\section{Peptides Derived from Structural Protein Digestion}

\section{Keratin-Based Peptides}

Topical formulations containing keratin-based peptides are described to have hemostatic, moisturizing, repair-promoting and potentially radio-protective properties. A placebo-controlled in vivo study (nine healthy females with dry skin types III to V) tested keratin-based peptide (molecular weight $<1000 \mathrm{Da})(3 \%)$ vs. deionized water $(3 \%)$ in a topical hand cream application which was compared to untreated areas. Biophysical results of keratin-based peptide treatment indicated skin improvement in water-holding capacity, hydration and elasticity. Results also indicated that the keratin-based peptide cream treatment can prevent some of the damaging effects associated with surfactant exposure [58]. Additional study results (six and 16 healthy females) with keratin based peptide (3\%) tested two different formulations (aqueous solution/internal wool lipid liposome suspension). It was shown that topically applied keratin based peptide formulations again improved the integrity and water-holding capacity of the skin barrier. Combining the keratin-based peptide with internal wool lipids led to additional beneficial effects [59]. The participant numbers were very low and future studies should be carried out with more volunteers. No detailed information on the mode of action of these peptides was available.

\section{Conclusions}

Twenty years ago, no scientist ever thought that the development or topical treatment of synthetic or natural related peptides for cosmetic purposes would be so effective. Researchers identified peptide sequences which allowed the peptides to be targeted to cosmetically important skin layers. Since 2000, the use of peptides in cosmeceutical products has increased dramatically, and this highlights the need to acquire in-depth knowledge of the different molecules as well as the physiological principles underlying their use. The commercial potential for these molecules is high, especially for those peptide sequences with cosmetic activity (e.g., anti-ageing, antioxidant, whitening). Nowadays, peptides can 
be developed or modified in numerous ways for solubility, better penetration, increased receptor activity, etc.

Selected peptides have excellent nontoxicity and stability records. Many of the studies shown in this review, and a variety of additional observations, clearly show the wide range of possible topical cosmetic applications of biologically active peptides, for improving the skin and its properties.

The overview also shows that there is still a lot of work required, and a lot of additional research to perform. While many synthetic peptides with confirmed efficacy in skin treatment have been researched to a satisfying extent, some of the mentioned clinical study results were obtained by using formulations containing peptides and other active ingredients (for example: niacinamide and vitamin C, E), or in the presence of a basic moisturizer. These clinical in vivo trials did not differentiate the role of the mentioned peptide specifically with other actives in the formulation. Hence, the observed results cannot be claimed clearly to be the effect of the used peptide for enhanced skin benefits and improved skin appearance.

Additionally, there are many more peptides that are used in cosmetics. Their clinical studies proving their efficacy are not frequently published in scientific journals. Some clinical studies were carried out with very few participant numbers, or through only a single study. These research results seem to be very promising, but for efficacy reasons, these peptides should be tested with a larger number of participants.

While the long-known and well-researched peptides like Cu-GHK are still undergoing research to obtain more details on their effectiveness and additional mechanisms of action, there are many other interesting peptides whose effects should be more closely investigated.

The reported beneficial clinical effects and the mode of action of a lot of peptides are not thoroughly explained.

Another aspect that hinders new innovations and peptide developments, is the non-existence of newly developed and lesser researched peptides on the Chinese INCI (International Nomenclature of Cosmetic Ingredients) list, presenting a significant barrier for entering the Chinese market.

There are still some open questions, such as how it may be possible to surmount the barrier to allow permeation of such compounds, and thereby enhance the concentrations delivered to the target site in the dermis. New questions will lead to new study designs, and hopefully new answers and solutions, and new cosmeceutical peptides.

Conflicts of Interest: The authors declare no conflict of interest.

\section{References}

1. Fields, K.; Falla, T.J.; Rodan, K.; Bush, L. Bioactive peptides: Signaling the future. J. Cosmet. Dermatol. 2009, 8, 8-13. [CrossRef] [PubMed]

2. Pai, V.V.; Bhandari, P.; Shukla, P. Topical peptides as cosmeceuticals. Indian J. Dermatol. Venereol. Leprol. 2017, 83, 9-18. [CrossRef] [PubMed]

3. Fischer, E.; Fourneau, E. Ueber einige derivate des Glykocolls. Eur. J. Inorg. Chem. 1901, 34, $2868-2877$. [CrossRef]

4. Wieland, T.; Bodanszky, M. The World of Peptides; Springer: Berlin/Heidelberg, Germany, 1991.

5. Rahnamaeian, M.; Vilcinskas, A. Short antimicrobial peptides as cosmetic ingredients to deter dermatological pathogens. Appl. Microbiol. Biotechnol. 2015, 99, 8847-8855. [CrossRef] [PubMed]

6. Gorouhi, F.; Maibach, H. Role of topical peptides in preventing or treating aged skin. Int. J. Cosmet. Sci. 2009, 31, 327-345. [CrossRef] [PubMed]

7. Mitragotri, S. Modeling skin permeability to hydrophilic and hydrophobic solutes based on four permeation pathways. J. Control. Release 2003, 86, 69-92. [CrossRef]

8. Partidos, C.D.; Beignon, A.S.; Brown, F.; Kramer, E.; Briand, J.P.; Muller, S. Applying peptide antigens onto bare skin: Induction of humoral and cellular immune responses and potential for vaccination. J. Control. Release 2002, 85, 27-34. [CrossRef] 
9. Lintner, K.; Peschard, O. Biologically active peptides: From a laboratory bench curiosity to a functional skin care product. Int. J. Cosmet. Sci. 2000, 22, 207-218. [CrossRef] [PubMed]

10. Kaczvinsky, J.R.; Griffiths, C.E.; Schnicker, M.S.; Li, J. Efficacy of anti-aging products for periorbital wrinkles as measured by 3-D imaging. J. Cosmet. Dermatol. 2009, 8, 228-233. [CrossRef] [PubMed]

11. Emanuele, E.; Spencer, J.M.; Braun, M. An experimental double-blind irradiation study of a novel topical product (TPF 50) compared to other topical products with DNA repair enzymes, antioxidants, and growth factors with sunscreens: Implications for preventing skin aging and cancer. J. Drugs Dermatol. 2014, 13, 309-314. [PubMed]

12. Nino, M.; Iovine, B.; Santoianni, P. Carnosine and N-Acetylcarnosine Induce Inhibition of UVB Erythema in Human Skin. J. Cosmet. Dermatol. Sci. Appl. 2011, 1, 177-179. [CrossRef]

13. Loing, E. Unipex Innovations, Québec, Canada; Thiery Suere and Elisabeth Lamarque, Unipex Innovations, Ramonville St. Agne, France, Trifluoroacetyl-Tripeptide-2 to Target Senescence for Anti-aging Benefits. Available online: http://www.cosmeticsandtoiletries.com/formulating/category/skincare/premiumTrifluoroacetyl-Tripept (accessed on 22 April 2017).

14. Puig, A.; Antón, J.M.; Mangues, M. A new decorin-like tetrapeptide for optimal organization of collagen fibres. Int. J. Cosmet. Sci. 2008, 30, 97-104. [CrossRef] [PubMed]

15. Safety Assessment of Palmitoyl Oligopeptides as Used in Cosmetics. Available online: http://www.cirsafety.org/sites/default/files/palmit072012slr.pdf (accessed on 22 April 2017).

16. Fournial, A.; Mondon, P. New Cosmetic or Dermopharmaceutical Topical Use of a Mixture of a Ghk Tripeptide and Gqpr Tetrapeptide. Available online: www.google.com/patents/WO2012164488A2?cl=en (accessed on 22 April 2017).

17. Murphy-Ullrich, J.E.; Poczatek, M. Activation of latent TGF-beta by thrombospondin-1: Mechanisms and physiology. Cytokine Growth Factor Rev. 2000, 11, 59-69. [CrossRef]

18. Imfeld, D.; Jackson, E.; Heidl, M.; Campiche, R. Activation of tgf a gateway to skin rejuvenation small synthetic-peptide mimics natural protein activity in skin to unlock tgf potential. HEPC Today-Househ. Pers. Care Today 2015, 10, 6-11.

19. Sanz, M.T.; Campos, C.; Milani, M.; Foyaca, M.; Lamy, A.; Kurdian, K.; Trullas, C. Biorevitalizing effect of a novel facial serum containing apple stem cell extract, pro-collagen lipopeptide, creatine, and urea on skin aging signs. J. Cosmet. Dermatol. 2016, 15, 24-30. [CrossRef] [PubMed]

20. Herndon, J.H., Jr.; Jiang, L.; Kononov, T.; Fox, T. An Open Label Clinical Trial of a Multi-Ingredient Anti-Aging Moisturizer Designed to Improve the Appearance of Facial Skin. J. Drugs Dermatol. 2015, 14, 699-704. [PubMed]

21. Lintner, K. Cosmetic or Dermopharmaceutical Use of Peptides for Healing, Hydrating and Improving Skin Appearances during Natural or Induced Ageing (Heliodermia, Pollution). US Pattent 6,620,419, 16 September 2003.

22. Robinson, L.R.; Fitzgerald, N.C.; Doughty, D.G.; Dawes, N.C.; Berge, C.A.; Bissett, D.L. Topical palmitoyl pentapeptide provides improvement in photoaged human facial skin. Int. J. Cosmet. Sci. 2005, 27, 155-160. [CrossRef] [PubMed]

23. Guttman, C. Studies demonstrate value of procollagen fragment Pal-KTTKS. Dermatol. Times 2002, 23, 68.

24. Mondon, P.; Hillion, M.; Peschard, O.; Andre, N.; Marchand, T.; Doridot, E.; Feuilloley, M.G.; Pionneau, C.; Chardonnet, S. Evaluation of dermal extracellular matrix and epidermal-dermal junction modifications using matrix-assisted laser desorption/ionization mass spectrometric imaging, in vivo reflectance confocal microscopy, echography, and histology: Effect of age and pepti. J. Cosmet. Dermatol. 2015, 14, 152-160. [CrossRef] [PubMed]

25. Watson, R.E.; Ogden, S.; Cotterell, L.F.; Bowden, J.J.; Bastrilles, J.Y.; Long, S.P.; Griffiths, C.E. Effects of a cosmetic 'anti-ageing' product improves photoaged skin. Br. J. Dermatol. 2009, 161, 419-426. [CrossRef] [PubMed]

26. Biopeptide EL ${ }^{\mathrm{TM}}$. Available online: http://www.webareal.com.ua/fotky27371/BIOPEPTIDE_EL_.pdf (accessed on 22 April 2017).

27. Rodrigues, A.L.; Benoit, I.; Clarius, T.; Pauly, G. Counter acting Ageing Phenomena by New Pure Tetrapeptides with Targeted Efficacy. Cosmet. Sci. Technol. 2009, 27, 63-71. 
28. Farwick, M.; Grether-Beck, S.; Marini, A.; Maczkiewitz, U.; Lange, J.; Köhler, T.; Lersch, P.; Falla, T.; Felsner, I.; Brenden, $\mathrm{H}$.; et al. Bioactive tetrapeptide GEKG boosts extracellular matrix formation: In vitro and in vivo molecular and clinical proof. Exp. Dermatol. 2011, 20, 602-604. [CrossRef] [PubMed]

29. Farwick, M.; Maczkiewitz, U.; Lersch, P.; Summers, B.; Rawlings, A.V. Facial skin-lightening benefits of the tetrapeptide Pro-Lys-Glu-Lys on subjects with skin types V-VI living in South Africa. J. Cosmet. Dermatol. 2011, 10, 217-223. [CrossRef] [PubMed]

30. Marini, A.; Farwick, M.; Grether-Beck, S.; Brenden, H.; Felsner, I.; Jaenicke, T.; Weber, M.; Schild, J.; Maczkiewitz, U.; Köhler, T.; et al. Modulation of skin pigmentation by the tetrapeptide PKEK: In vitro and in vivo evidence for skin whitening effects. Exp. Dermatol. 2012, 21, 140-146. [CrossRef] [PubMed]

31. Gruber, J.V.; Ludwig, P.; Holtz, R. Modulation of cellular senescence in fibroblasts and dermal papillae cells in vitro. J. Cosmet. Sci. 2013, 64, 79-87. [PubMed]

32. Linder, J. The science behind peptides. Plast. Surg. Nurs. 2012, 32, 71-72. [CrossRef] [PubMed]

33. Pickart, L.; Schagen, S. New data of the Cosmeceutical and tripeptide GHK. SOFW J. 2015, 9, 141.

34. Campbell, J.D.; McDonough, J.E.; Zeskind, J.E.; Hackett, T.L.; Pechkovsky, D.V.; Brandsma, C.A.; Suzuki, M.; Gosselink, J.V.; Liu, G.; Alekseyev, Y.O.; et al. A gene expression signature of emphysema-related lung destruction and its reversal by the tripeptide GHK. Genome Med. 2012, 4, 67. [PubMed]

35. Kerscher, M.; Buntrock, A. Update in der dermatologischen Kosmetik (Update on cosmeceuticals). JDDG 2011, 9, 314-328. [PubMed]

36. Leyden, J.J.; Stevens, T.; Finkey, M.B.; Barkovic, S. Skin care benefits of copper peptide containing facial cream. In Proceedings of the American Academy of Dermatology 60th Annual Meeting, New Orleans, LA, USA, 22-27 February 2002; p. 29.

37. Pickart, L.; Vasquez-Soltero, J.M.; Margolina, A. GHK Peptide as a Natural Modulator of Multiple Cellular Pathways in Skin Regeneration. BioMed Res. Int. 2015, 2015. [CrossRef] [PubMed]

38. Jose, S.; Hughbanks, M.L.; .Binder, B.Y.; Ingavle, G.C.; Leach, J.K. Enhanced trophic factor secretion by mesenchymal stem/stromal cells with Glycine-Histidine-Lysine (GHK)-modified alginate hydrogels. Acta Biomater. 2014, 10, 1955-1964. [CrossRef] [PubMed]

39. Abdulghani, A.; Sherr, A.; Shirin, S.; Solodkina, G.; Tapia, E.; Wolf, B.; Gottlieb, A. Effects of topical creams containing vitamin $\mathrm{C}$, a copper-binding peptide cream and melatonin compared with tretinoin on the ultrastructure of normal skin-A pilot clinical, histologic, and ultrastructural study. Dis. Manag. Clin. Outcomes 1998, 1, 136-141. [CrossRef]

40. Finkley, M.; Appa, Y.; Bhandarkar, S. Copper Peptide and Skin Cosmeceuticals and Active Cosmetics: Drugs vs. Cosmetics; Elsner, P., Maibach, H., Eds.; Marcel Dekker: New York, NY, USA, 2005; pp. 549-563.

41. Hussain, M.; Goldberg, D.J. Topical manganese peptide in the treatment of photodamaged skin. J. Cosmet. Laser Ther. 2007, 9, 232-236. [CrossRef] [PubMed]

42. Ruiz, M.A.; Clares, B.; Morales, M.E.; Cazalla, S.; Gallardo, V. Preparation and stability of cosmetic formulations with an anti-aging peptide. J. Cosmet. Sci. 2007, 58, 157-171. [CrossRef] [PubMed]

43. Krishnan, G.; Roberts, M.S.; Grice, J.; Anissimov, Y.G.; Moghimi, H.R.; Benson, H.A. Iontophoretic skin permeation of peptides: An investigation into the influence of molecular properties, iontophoretic conditions and formulation parameters. Drug Deliv. Transl. Res. 2014, 4, 222-232. [CrossRef] [PubMed]

44. Blanes-Mira, C.; Clemente, J.; Jodas, G.; Gil, A.; Fernández-Ballester, G.; Ponsati, B.; Gutierrez, L.; Pérez-Payá, E.; Ferrer-Montiel, A. A synthetic hexapeptide (Argireline) with antiwrinkle activity. Int. J. Cosmet. Sci. 2002, 24, 303-310. [CrossRef] [PubMed]

45. Wang, Y.; Wang, M.; Xiao, S.; Pan, P.; Li, P.; Huo, J. The anti-wrinkle efficacy of argireline, a synthetic hexapeptide, in Chinese subjects: A randomized, placebo-controlled study. Am. J. Clin. Dermatol. 2013, 14, 147-153. [CrossRef] [PubMed]

46. Zhmak, M.N.; Utkin, Y.N.; Andreeva, T.V.; Kudryavtsev, D.S.; Kryudova, E.V.; Tsetlin, V.I.; Shelukhina, I.V.E. Peptide Inhibitors of Nicotinic Acetylcholine Receptor. US Patent US 20,150,361,137 A1, 17 December 2015.

47. Leuphasyl a New Pentapeptide for Expression Wrinkles. Available online: http://docplayer.net/15401907Leuphasyl-a-new-pentapeptide-for-expression-wrinkles-code-pd080-date-june-2005-revision-1-a-gmppeptide-for-cosmetic-applications.html (accessed on 19 December 2016).

48. Dragomirescu, A.O.; Andoni, M.; Ionescu, D.; Andrei, F. The Efficiency and Safety of Leuphasyl-A Botox-Like Peptide. Cosmetics 2014, 1, 75-81. [CrossRef] 
49. Rull, M.; Davi, C.; Cañadas, E.; Almiñana, N.; Delgado, R. Peptide Approach to Enhance Anti-wrinkle Efficacy Between Injections. Available online: http://www.cosmeticsandtoiletries.com/formulating/ category/antiaging/Peptide-Approach-to-Enhance-Anti-wrinkle-Efficacy-Between-Injections-premium273052431.html (accessed on 15 May 2017).

50. Chhipa, N.M.R.; Chaudhari, B. Toxin as a Medicine. Available online: https://www.researchgate.net/profile/ Nadim_Chhipa/publication/269037373_TOXIN_AS_A_MEDICINE/links/547dfbf70cf241bf4b5b9b40.pdf (accessed on 22 April 2017).

51. Trookman, N.S.; Rizer, R.L.; Ford, R.; Ho, E.; Gotz, V. Immediate and Long-term Clinical Benefits of a Topical Treatment for Facial Lines and Wrinkles. J. Clin. Aesthet. Dermatol. 2009, 2, 38-43. [PubMed]

52. Zhou, B.R.; Ma, L.W.; Liu, J.; Zhang, J.A.; Xu, Y.; Wu, D.; Permatasari, F.; Luo, D. Protective Effects of Soy Oligopeptides in Ultraviolet B-Induced Acute Photodamage of Human Skin. Oxid. Med. Cell. Longev. 2016, 2016. [CrossRef] [PubMed]

53. Andre-Frei, V.; Perrier, E.; Augustin, C.; Damour, O.; Bordat, P.; Schumann, K.; Förster, T.; Waldmann-Laue, M. A comparison of biological activities of a new soya biopeptide studied in an in vitro skin equivalent model and human volunteers. Int. J. Cosmet. Sci. 1999, 21, 299-311. [CrossRef] [PubMed]

54. Kim, D.W.; Hwang, H.S.; Kim, D.S.; Sheen, S.H.; Heo, D.H.; Hwang, G.; Kang, S.H.; Kweon, H.; Jo, Y.Y.; Kang, S.W.; et al. Effect of silk fibroin peptide derived from silkworm Bombyx mori on the anti-inflammatory effect of Tat-SOD in a mice edema model. BMB Rep. 2011, 44, 787-792. [CrossRef] [PubMed]

55. Sim, G.S.; Lee, D.H.; Kim, J.H.; An, S.K.; Choe, T.B.; Kwon, T.J.; Pyo, H.B.; Lee, B.C. Black rice (Oryza sativa L. var. japonica) hydrolyzed peptides induce expression of hyaluronan synthase 2 gene in HaCaT keratinocytes. J. Microbiol. Biotechnol. 2007, 17, 271-279. [PubMed]

56. Ochiai, A.; Tanaka, S.; Tanaka, T.; Taniguchi, M. Rice bran protein as a potent source of Antimelanogenic peptides with tyrosinase inhibitory activity. J. Nat. Prod. 2016, 79, 2545-2551. [CrossRef] [PubMed]

57. Manosroi, A.; Chutoprapat, R.; Abe, M.; Manosroi, W.; Manosroi, J. Anti-aging efficacy of topical formulations containing niosomes entrapped with rice bran bioactive compounds. Pharm. Biol. 2012, 50, $208-224$. [CrossRef] [PubMed]

58. Barba, C.; Méndez, S.; Roddick-Lanzilotta, A.; Kelly, R.; Parra, J.L.; Coderch, L. Wool peptide derivatives for hand care. J. Cosmet. Sci. 2007, 58, 99-107. [CrossRef] [PubMed]

59. Barba, C.; Méndez, S.; Roddick-Lanzilotta, A.; Kelly, R.; Parra, J.L.; Coderch, L. Cosmetic effectiveness of topically applied hydrolysed keratin peptides and lipids derived from wool. Skin Res Technol. 2008, 14, 243-248. [CrossRef] [PubMed] 